\title{
A PECULIARITY OF THE CREATION OPERATOR*,†
}

\author{
JAN STOCHEL and FRANCISZEK HUGON SZAFRANIEC \\ Instytut Matematyki, Uniwersytet Jagielloński, ul. Reymonta 4, PL-30059 Kraków, Poland \\ e-mail: stochel@im.uj.edu.pl andfhszafra@im.uj.edu.pl
}

(Received 24 May, 2000; accepted 25 August 2000)

\begin{abstract}
It is shown that the creation operator is the only (up to a multiplicative constant) injective weighted shift all of whose translations (or at least one) are still injective weighted shifts regardless of what the weight sequences and the bases are. A similar result is true for the annihilation operator as well as for the Heisenberg and Schrödinger couples.
\end{abstract}

2000 Mathematics Subject Classification. Primary 47B37, 81S05; Secondary 47B15, 47B20, 47B47.

0. Introduction. The creation operator

$$
a^{+}=\frac{1}{\sqrt{2}}\left(x-\frac{d}{d x}\right),
$$

the classical object of quantum mechanics ${ }^{1}$, has the following remarkable property: for every complex number $\lambda$, the operator $a^{+}+\lambda I$ is unitarily equivalent to $a^{+}$. This observation has been exploited by the second named author in a series of papers $[17,18,19,20]$, where various models for the creation operator have been invented. (For applications of this idea in physics see $[3,4]$.) If we look at the creation operator as the weighted shift with respect to the orthonormal basis composed of Hermite functions, then we can say that for every complex number $\lambda, a^{+}+\lambda I$ is a weighted shift, but with respect to a different basis. A natural question arises whether this invariance property distinguishes the creation operator among all weighted shifts. In this paper we show that this is the case. Even more, it turns out to be sufficient to assume that at least one nonzero translation of a weighted shift is a weighted shift.

The creation operator together with its (at least formally) adjoint, the annihilation one, defined as

$$
a^{-}=\frac{1}{\sqrt{2}}\left(x+\frac{d}{d x}\right)
$$

satisfy the commutation relation

$$
a^{-} a^{+}-a^{+} a^{-}=I
$$

*Dedicated to the memory of I. E. Segal.

${ }^{\dagger}$ The research resulting in this paper was supported at its final stage by the KBN grant \#2 P03A 00417.

${ }^{1}$ Let us recommend here, by the way, a beautiful overview [9] of the story of the quantum harmonic oscillator written by mathematicians. 
Because those two operators are the only solutions (under some circumstances $[23,11,21])$ of the aforesaid commutation relation we may say that our result shows how spatial properties force algebraic ones to hold. The same somewhat philosophical remark refers to the Heisenberg couple.

Given a linear operator $A$ in a (complex) Hilbert space $\mathcal{H}$, we denote by $\mathcal{D}(A)$ the domain of $A$, by $\mathcal{N}(A)$ the kernel of $A$ and by $A^{*}$ the adjoint of $A$. In case $A$ is closable, we write $\bar{A}$ for the closure of $A$. A linear subspace $\mathcal{E}$ of $\mathcal{D}(A)$ is said to be a core for a closable operator $A$ if $\bar{A}=\left(\left.A\right|_{\mathcal{E}}\right)^{-}$, where $\left.A\right|_{\mathcal{E}}$ stands for the restriction of $A$ to $\mathcal{E}$. By $\boldsymbol{B}(\mathcal{H})$ we understand the $C^{*}$-algebra of all bounded linear operators on $\mathcal{H}$; $I$ stands for the identity operator on $\mathcal{H}$. Denote by $\operatorname{lin} \mathcal{F}$ the linear span of a subset $\mathcal{F}$ of $\mathcal{H}$.

1. Weighted shifts. Suppose $S$ is a closable densely defined linear operator in a separable Hilbert space $\mathcal{H}$. We say that $S$ is a weighted shift $t^{2}$ if there exists an orthonormal basis $\boldsymbol{e}=\left\{e_{n}\right\}_{n=0}^{\infty}$ for $\mathcal{H}$ and a sequence $\left\{\sigma_{n}\right\}_{n=0}^{\infty}$ of nonzero complex numbers such that $\mathcal{D}_{e}=\operatorname{lin}\left\{e_{n} ; n \geq 0\right\}$ is a core for $S$ and $S e_{k}=\sigma_{k} e_{k+1}$ for $k \geq 0$. If this happens, then $S$ is said to be a weighted shift with respect to the basis $\boldsymbol{e}$, and with weights $\left\{\sigma_{n}\right\}_{n=0}^{\infty}$. Notice that $\bar{S}=V D$, where $V$ is a unique linear isometry on $\mathcal{H}$ such that $V e_{n}=e_{n+1}$ for $n \geq 0$ and $D$ is a unique normal operator in $\mathcal{H}$ such that $D e_{n}=\sigma_{n} e_{n}$ for $n \geq 0$. Consequently $\bar{S}$ is injective, $S^{*}=D^{*} V^{*}$ and

$$
\begin{gathered}
\mathcal{D}(\bar{S})=\left\{f \in \mathcal{H} ; \sum_{n=0}^{\infty}\left|\left\langle f, e_{n}\right\rangle \sigma_{n}\right|^{2}<\infty\right\}, \\
\bar{S} f=\sum_{n=0}^{\infty}\left\langle f, e_{n}\right\rangle \sigma_{n} e_{n+1}, \quad f \in \mathcal{D}(\bar{S}), \\
\mathcal{D}\left(S^{*}\right)=\left\{f \in \mathcal{H} ; \sum_{n=0}^{\infty}\left|\left\langle f, e_{n+1}\right\rangle \sigma_{n}\right|^{2}<\infty\right\}, \\
S^{*} f=\sum_{n=0}^{\infty}\left\langle f, e_{n+1}\right\rangle \bar{\sigma}_{n} e_{n}, \quad f \in \mathcal{D}\left(S^{*}\right) .
\end{gathered}
$$

In particular, we have $\mathcal{D}_{\boldsymbol{e}} \subset \mathcal{D}\left(S^{*}\right), S^{*} e_{0}=0$ and $S^{*} e_{k+1}=\bar{\sigma}_{k} e_{k}$ for $k \geq 0$.

Notice that any two closed weighted shifts with the same weights are unitarily equivalent. Indeed, if $S_{j}$ is a closed weighted shift (acting in a Hilbert space $\mathcal{H}_{j}$ ) with respect to an orthonormal basis $\left\{e_{j, n}\right\}_{n=0}^{\infty}$, and with weights $\left\{\sigma_{n}\right\}_{n=0}^{\infty}, j=1,2$, then $U S_{1}=S_{2} U$, where $U: \mathcal{H}_{1} \rightarrow \mathcal{H}_{2}$ is a unique unitary isomorphism such that $U e_{1, n}=e_{2, n}$ for $n \geq 0$ (use (1)).

Weighted shifts appear on many occasions; usually one starts with a basis with respect to which an operator is a weighted shift and keeps the basis fixed afterwards and this is, maybe, why the following simple question has not attracted any attention. The question is: can an operator be a weighted shift with respect to different

$\overline{2}[\mathbf{1 3}]$ may serve as a first introduction of the bounded case; for unbounded weighted shifts see [6], [15] and $[10]$. 
bases, and with different weights or, in other words, how does the definition depend on choice of an orthonormal basis? In the context of the present paper this question is natural; the answer is in the following result.

Proposition 1. Suppose that a linear operator $S$ in $\mathcal{H}$ is a weighted shift with respect to an orthonormal basis $\left\{e_{i, n}\right\}_{n=0}^{\infty}$ with weights $\left\{\sigma_{i, n}\right\}_{n=0}^{\infty}$, where $i=1,2$. Then there exists a sequence $\left\{\gamma_{n}\right\}_{n=0}^{\infty}$ of complex numbers such that

$$
\begin{aligned}
e_{2, n} & =\gamma_{n} e_{1, n} & & n \geq 0, \\
\left|\sigma_{1, n}\right| & =\left|\sigma_{2, n}\right| & & n \geq 0 .
\end{aligned}
$$

Proof. Set $\sigma_{1,-1}=0$ and $e_{1,-1}=0$. Since $S^{*} e_{1, k}=\bar{\sigma}_{1, k-1} e_{1, k-1}$ for $k \geq 0$, we get

$$
\begin{aligned}
\sigma_{2, n}\left\langle e_{2, n+1}, e_{1, k}\right\rangle=\left\langle S e_{2, n}, e_{1, k}\right\rangle & =\left\langle e_{2, n}, S^{*} e_{1, k}\right\rangle \\
& =\sigma_{1, k-1}\left\langle e_{2, n}, e_{1, k-1}\right\rangle \quad(k, n \geq 0) .
\end{aligned}
$$

This leads to $\left\langle e_{2, n}, e_{1,0}\right\rangle=0$ for $n \geq 1$ and

$$
\left\langle e_{2, n+1}, e_{1, k+1}\right\rangle=\frac{\sigma_{1, k}}{\sigma_{2, n}}\left\langle e_{2, n}, e_{1, k}\right\rangle \quad(k, n \geq 0) .
$$

Hence

$$
\left\langle e_{2, n}, e_{1, k}\right\rangle=0 \quad(n \geq k+1) .
$$

The proof of (3) is by induction on $n$. It follows from (6) that $e_{1,0}$ belongs to the orthogonal complement of the set $\left\{e_{2, m} ; m \geq 1\right\}$ and so $e_{1,0} \in \mathbb{C} \cdot e_{2,0}$. If $e_{1, j} \in \mathbb{C} \cdot e_{2, j}$ for $j=0, \ldots, n$, then by (6) the vector $e_{1, n+1}$ belongs to the orthogonal complement of the set $\left\{e_{2, m} ; m \neq n+1\right\}$. Hence $e_{1, n+1} \in \mathbb{C} \cdot e_{2, n+1}$. Finally, condition (4) is a consequence of (5). This completes the proof.

Consider an arbitrary weighted shift $S$ with respect to an orthonormal basis $\left\{e_{n}\right\}_{n=0}^{\infty}$ with weights $\left\{\sigma_{n}\right\}_{n=0}^{\infty}$. Set $\gamma_{n}=\sigma_{0} \sigma_{1} \cdots \sigma_{n-1}\left|\sigma_{0} \sigma_{1} \cdots \sigma_{n-1}\right|^{-1}$ for $n \geq 1$ and $\gamma_{0}=1$. Then one can check that $S$ is a weighted shift with respect to a new orthonormal basis $\left\{\gamma_{n} e_{n}\right\}_{n=0}^{\infty}$, and with new weights $\left\{\left|\sigma_{n}\right|\right\}_{n=0}^{\infty}$. In other words, for any weighted shift $S$ we can always find an orthonormal basis with respect to which $S$ has positive weights.

2. The translation invariance property. By an (abstract) creation operator in a Hilbert space $\mathcal{H}$ we understand here a closed weighted shift with weights $\{\sqrt{n+1}\}_{n=0}^{\infty}$. We infer from what we have mentioned in Section 1 that, in particular, every two creation operators are unitarily equivalent. The basic property of a creation operator $S$ is that all its translations $S-\lambda I, \lambda \in \mathbb{C}$, are still creation operators. This follows from the commutation relation ${ }^{3}$

$$
(S-\lambda I)^{*}(S-\lambda I)=(S-\lambda I)(S-\lambda I)^{*}+I \quad(\lambda \in \mathbb{C}),
$$

${ }^{3}$ The relation (7) is a direct consequence of $S^{*} S=S S^{*}+I$; the latter equality can be proved with the help of (1) and (2). 
the fact that the closed operator $S-\lambda I$ is irreducible (cf. [6, Section 3]) and Tillmann's theorem (cf. [23], [11, Theorem 4.4.1] or, for an approach involving subnormality, [21]). Explicit orthonormal bases with respect to which $S+\lambda I, \lambda>0$, are creation operators are constructed in $[\mathbf{1 7 , 1 9 ]}$. (Some related constructions are in $[\mathbf{1 8 , 2 0}]$.) In the proof of Theorem 4 below we propose something which can be viewed as an abstract way of generating such bases. Our method does not appeal to the spectral theorem.

According to what we have said so far a creation operator $S$ has the property that each of its translations $S-\lambda I$ remains in the class of weighted shifts and is unitarily equivalent to $S$. Though this invariance property holds, as we show below, also for other classes of operators, within the class of weighted shifts it happens only for the creation operator, and this (Theorem 4) is the main conclusion of this paper.

The example that follows is of a normal operator $N$ which is unitarily equivalent to $N-\lambda I$ for every $\lambda \in \mathbb{C}$. (Recall that a creation operator is subnormal and irreducible.)

ExAmple 2. Let $\mathcal{H}$ be the Hilbert space of all complex Borel functions on $\mathbb{C}$ that are square summable with respect to the planar Lebesgue measure $\mu$ and let $\mathbb{C} \ni \lambda \rightarrow U_{\lambda} \in \boldsymbol{B}(\mathcal{H})$ be the unitary group of translations; i.e.

$$
\left(U_{\lambda} f\right)(z)=f(z-\lambda), \quad z \in \mathbb{C}, f \in \mathcal{H} .
$$

Denote by $N$ the operator of multiplication by " $z$ " in $\mathcal{H}$, i.e., $(N f)(z)=z f(z)$ for $z \in \mathbb{C}$ and $f \in \mathcal{H}$. Then $(N-\lambda I) U_{\lambda}=U_{\lambda} N$, which means that the normal operators $N$ and $N-\lambda I$ are unitarily equivalent for every $\lambda \in \mathbb{C}$.

The above considerations lead to the following question. Under what circumstances is a closed densely defined operator $A$ unitarily equivalent to $A+\lambda I$ ? ( $\lambda$ is a fixed nonzero complex number.) In case $A$ is a generator of a $\mathcal{C}_{0}$-semigroup, the answer is given below.

Proposition 3. Let $A$ be an infinitesimal generator of a $\mathcal{C}_{0}$-semigroup $\{T(t) ; t>0\} \subset \boldsymbol{B}(\mathcal{H})$ and let $\lambda$ be a complex number. If $U \in \boldsymbol{B}(\mathcal{H})$ is a unitary operator, then the following conditions are equivalent:

(i) $(A+\lambda I) U=U A$,

(ii) $U T(t)=e^{\lambda t} T(t) U$ for every $t>0$.

Proof. Assuming (i) we get $U \mathcal{D}(A) \subset \mathcal{D}(A)$ and $(U A-A U) h=\lambda U h$, for $h \in \mathcal{D}(A)$, so that (ii) is a direct consequence of [8, Part I, Proposition 1.0].

If (ii) holds, then by [8, Part I, Proposition 1.0] we have $U \mathcal{D}(A) \subset \mathcal{D}(A)$ and $(U A-A U) h=\lambda U h$, for $h \in \mathcal{D}(A)$. This is equivalent to

$$
U A \subset(A+\lambda I) U
$$

However $U$ is unitary, and so $U^{*} T(t)=e^{-\lambda t} T(t) U^{*}$, for all $t>0$. Applying once more [8, Part I, Proposition 1.0], we get $U^{*} \mathcal{D}(A) \subset \mathcal{D}(A)$ which, together with the inclusion (8), yields (i).

Let $A, T(\cdot)$ and $\lambda$ be as in Proposition 3. Following Mlak [8, Part I], we say that an operator $X \in \boldsymbol{B}(\mathcal{H})$ is of class $(T(\cdot), \lambda)$ if $X T(t)=e^{\lambda t} T(t) X$ for every $t>0$; if, 
moreover, $A=i S$ and $\lambda=i \alpha$, where $S$ is a selfadjoint operator and $\alpha$ is a nonzero real number, then $X$ is called $(S, \alpha)$-circular. By Proposition 3, an infinitesimal generator $A$ of a $\mathcal{C}_{0}$-semigroup $T(\cdot)$ is unitarily equivalent to $A+\lambda I$ if and only if there exists a unitary operator of class $(T(\cdot), \lambda)$. This immediately excludes the existence of unitary operators of class $(T(\cdot), \lambda)$ in case the spectrum $\operatorname{sp}(A)$ of $A$ is in a "wrong" position with respect to $\lambda$. For example, if $S$ is a semibounded selfadjoint operator and $\alpha \in \mathbb{R} \backslash\{0\}$, then there is no unitary $(S, \alpha)$-circular operator. Indeed, otherwise by Proposition 3 the operators $S$ and $S+\alpha I$ are unitarily equivalent, so that $\operatorname{sp}(S)=\operatorname{sp}(S)+\alpha$, which is impossible due to the fact that $\operatorname{sp}(S)$ is a nonempty semibounded subset of $\mathbb{R}$. (Our proof is much shorter than that of [8, Part I, Theorem 2.1].) However, it may happen that the spectrum of $A$ is "better" located with respect to $\lambda$, but still there is no unitary operator of class $(T(\cdot), \lambda)(\mathrm{cf}$. $[8$, Part I, Corollary 2.0]). We refer the reader to $[\mathbf{1}],[\mathbf{5 , 7 , 8}]$ and $[\mathbf{2 2}]$ for more details concerning circular operators (including unitary ones).

3. Characterization of the creation operator. Below we characterize the creation operator (up to a multiplicative constant) as a closed weighted shift all of whose translations are still weighted shifts. Before this we would like to encourage the reader to look at the definition of weighted shifts once more.

TheOREM 4. Let $S$ be a closed weighted shift in a Hilbert space $\mathcal{H}$. Then the following conditions are equivalent:

(i) for every $\lambda \in \mathbb{C}, S-\lambda I$ is a weighted shift,

(ii) there exists $\lambda \in \mathbb{C} \backslash\{0\}$ such that $S-\lambda I$ is a weighted shift,

(iii) there exists $\alpha \in \mathbb{C} \backslash\{0\}$ such that $\alpha S$ is a creation operator.

Moreover, if $S$ is a creation operator, then $S-\lambda I$ is a creation operator for every $\lambda \in \mathbb{C}$.

Proof. (ii) $\Rightarrow$ (iii) Suppose $S$ is a weighted shift with respect to an orthonormal basis $\left\{e_{n}\right\}_{n=0}^{\infty}$ with weights $\left\{\sigma_{n}\right\}_{n=0}^{\infty}$ and, for some $\lambda \neq 0, S-\lambda I$ is a weighted shift with respect to an orthonormal basis $\left\{f_{n}\right\}_{n=0}^{\infty}$ with weights $\left\{\tau_{n}\right\}_{n=0}^{\infty}$. Without any loss of generality we can assume that $\sigma_{n}>0$ and $\tau_{n}>0$ for $n \geq 0$. Since $S-\lambda I$ is a weighted shift with respect to $\left\{f_{n}\right\}_{n=0}^{\infty}$, we get $(S-\lambda I)^{*} f_{0}=0$ or equivalently $S^{*} f_{0}=\bar{\lambda} f_{0}$. However $S$ is a weighted shift with respect to $\left\{e_{n}\right\}_{n=0}^{\infty}$ and so, by the description (2) of $S^{*}$, we have

$$
\sum_{n=0}^{\infty}\left\langle f_{0}, e_{n+1}\right\rangle \sigma_{n} e_{n}=S^{*} f_{0}=\sum_{n=0}^{\infty} \bar{\lambda}\left\langle f_{0}, e_{n}\right\rangle e_{n} .
$$

This in turn implies that $\left\langle f_{0}, e_{n+1}\right\rangle=\left\langle f_{0}, e_{n}\right\rangle \bar{\lambda} \sigma_{n}^{-1}$ for $n \geq 0$, and hence that

$$
\left\langle f_{0}, e_{n}\right\rangle=\frac{\bar{\lambda}^{n}}{\sigma_{0} \cdots \sigma_{n-1}}\left\langle f_{0}, e_{0}\right\rangle, \quad n \geq 1 .
$$

Set $a=\left\langle f_{0}, e_{0}\right\rangle$. Since $f_{0} \neq 0$, we conclude from (9) that $a \neq 0$. Summarizing, we have proved the following equation ${ }^{4}$ :

${ }^{4}$ The equality (10) can be found in [14, Section 16] as a consequence of much more general results; here we have included its short and direct proof, just for reader's convenience. 


$$
f_{0}=a\left(e_{0}+\sum_{n=1}^{\infty} \frac{\bar{\lambda}^{n}}{\sigma_{0} \cdots \sigma_{n-1}} e_{n}\right)
$$

As $S-\lambda I$ is a weighted shift with respect to $\left\{f_{n}\right\}_{n=0}^{\infty}$, we get

$$
(S-\lambda I)^{*}(S-\lambda I) f_{0}=\tau_{0}^{2} f_{0}=a \tau_{0}^{2}\left(e_{0}+\sum_{n=1}^{\infty} \frac{\bar{\lambda}^{n}}{\sigma_{0} \cdots \sigma_{n-1}} e_{n}\right) .
$$

We know that $f_{0} \in \mathcal{D}\left((S-\lambda I)^{*}\right)=\mathcal{D}\left(S^{*}\right)$ and $S f_{0}-\lambda f_{0}=\tau_{0} f_{1} \in \mathcal{D}\left(S^{*}\right)$. Hence $S f_{0} \in \mathcal{D}\left(S^{*}\right)$, which means that $f_{0} \in \mathcal{D}\left(S^{*} S\right) \cap \mathcal{D}\left(S^{*}\right)$. This and (10) lead via (1) and (2) to

$$
\begin{aligned}
(S-\lambda I)^{*}(S-\lambda I) f_{0} & =S^{*} S f_{0}-\lambda S^{*} f_{0}-\bar{\lambda} S f_{0}+|\lambda|^{2} f_{0}=S^{*} S f_{0}-\bar{\lambda} S f_{0} \\
& =a\left(S^{*}-\bar{\lambda}\right)\left(\sigma_{0} e_{1}+\sum_{n=1}^{\infty} \frac{\overline{\lambda^{n}} \sigma_{n}}{\sigma_{0} \cdots \sigma_{n-1}} e_{n+1}\right) \\
& =a\left(\left(\sigma_{0}^{2} e_{0}+\sum_{n=1}^{\infty} \frac{\bar{\lambda}^{n} \sigma_{n}^{2}}{\sigma_{0} \cdots \sigma_{n-1}} e_{n}\right)-\left(\bar{\lambda} \sigma_{0} e_{1}+\sum_{n=2}^{\infty} \frac{\bar{\lambda}^{n} \sigma_{n-1}}{\sigma_{0} \cdots \sigma_{n-2}} e_{n}\right)\right) \\
& =a\left(\sigma_{0}^{2} e_{0}+\bar{\lambda}\left(\frac{\sigma_{1}^{2}}{\sigma_{0}}-\sigma_{0}\right) e_{1}+\sum_{n=2}^{\infty} \frac{\bar{\lambda}^{n}}{\sigma_{0} \cdots \sigma_{n-2}}\left(\frac{\sigma_{n}^{2}}{\sigma_{n-1}}-\sigma_{n-1}\right) e_{n}\right),
\end{aligned}
$$

which together with (11) gives us (recall that $\lambda \neq 0$ )

$$
\sigma_{n}^{2}-\sigma_{n-1}^{2}=\tau_{0}^{2}=\sigma_{0}^{2}, \quad n \geq 1 .
$$

Thus $\sigma_{n}=\sigma_{0} \sqrt{n+1}$ for $n \geq 0$, which means that $\sigma_{0}^{-1} S$ is a creation operator with respect to $\left\{e_{n}\right\}_{n=0}^{\infty}$.

(iii) $\Rightarrow$ (i) Without any loss of generality we can assume that $\alpha=1$. Suppose $S$ is a creation operator with respect to an orthonormal basis $\boldsymbol{e}=\left\{e_{n}\right\}_{n=0}^{\infty}$. Set

$$
\mathcal{D}^{\infty}\left(S, S^{*}\right)=\bigcap\left\{\mathcal{D}\left(S_{1} \ldots S_{n}\right) ; S_{1}, \ldots, S_{n} \in\left\{S, S^{*}\right\}, n \geq 1\right\}
$$

$S_{\lambda}=S-\lambda I$ and $S_{\lambda}^{*}=\left(S_{\lambda}\right)^{*}$, for $\lambda \in \mathbb{C}$. Then $\mathcal{D}^{\infty}\left(S, S^{*}\right)$ is the largest linear subspace of $\mathcal{D}(S) \cap \mathcal{D}\left(S^{*}\right)$ that is invariant for $S$ and $S^{*}$, and which contains $\mathcal{D}_{\boldsymbol{e}}$. It follows from (7) that $\left(S_{\lambda}^{*} S_{\lambda}-S_{\lambda} S_{\lambda}^{*}\right) h=h$, for $h \in \mathcal{D}^{\infty}\left(S, S^{*}\right)$. Thus, by [16, Lemma], we have

$$
S_{\lambda}^{* i} S_{\lambda}^{j} h=\sum_{k=0}^{\infty} k !\left(\begin{array}{l}
i \\
k
\end{array}\right)\left(\begin{array}{l}
j \\
k
\end{array}\right) S_{\lambda}^{j-k} S_{\lambda}^{*(i-k)} h, \quad h \in \mathcal{D}^{\infty}\left(S, S^{*}\right), \quad(i, j \geq 0),
$$

where, by definition, $S_{\lambda}^{n}=S_{\lambda}^{* n}=0$ for $n<0$ and $\left(\begin{array}{c}n \\ m\end{array}\right)=0$ for $n<m$. Set

$$
f_{0}(\lambda)=e^{-|\lambda|^{2} / 2} \sum_{n=0}^{\infty} \frac{\bar{\lambda}^{n}}{\sqrt{n !}} e_{n}, \quad \lambda \in \mathbb{C} .
$$

Then $f_{0}(\lambda) \in \mathcal{D}\left(S_{\lambda}^{*}\right), S_{\lambda}^{*} f_{0}(\lambda)=0$ and $\left\|f_{0}(\lambda)\right\|=1$ (cf. [10, $\left.\S 1\right]$ ). One can deduce from (13) via (1) and (2) that $f_{0}(\lambda) \in \mathcal{D}^{\infty}\left(S, S^{*}\right)=\mathcal{D}^{\infty}\left(S_{\lambda}, S_{\lambda}^{*}\right)$. This implies that $f_{n}(\lambda) \stackrel{\text { df }}{=} \frac{1}{\sqrt{n !}} S_{\lambda}^{n} f_{0}(\lambda) \in \mathcal{D}^{\infty}\left(S, S^{*}\right)$ for $n \geq 1$. Since $S_{\lambda}^{*} f_{0}(\lambda)=0$, (12) yields 


$$
\left\langle f_{j}(\lambda), f_{i}(\lambda)\right\rangle=\sum_{k=0}^{\infty} \frac{k !}{\sqrt{i ! j !}}\left(\begin{array}{l}
i \\
k
\end{array}\right)\left(\begin{array}{l}
j \\
k
\end{array}\right)\left\langle S_{\lambda}^{*(i-k)} f_{0}(\lambda), S_{\lambda}^{*(j-k)} f_{0}(\lambda)\right\rangle=\delta_{i, j} \quad i, j \geq 0 .
$$

This means that $\left\{f_{n}(\lambda)\right\}_{n=0}^{\infty}$ is an orthonormal sequence in $\mathcal{H}$ and

$$
S_{\lambda} f_{n}(\lambda)=\sqrt{n+1} f_{n+1}(\lambda) \quad n \geq 0, \lambda \in \mathbb{C} .
$$

Applying once more (12), we get

$$
S_{\lambda}^{*} f_{0}(\lambda)=0 \quad \& \quad S_{\lambda}^{*} f_{n+1}(\lambda)=\sqrt{n+1} f_{n}(\lambda) \quad(n \geq 0) .
$$

Set $\mathcal{E}_{\lambda}=\operatorname{lin}\left\{f_{n}(\lambda) ; n \geq 0\right\}=\operatorname{lin}\left\{S^{n} f_{0}(\lambda) ; n \geq 0\right\}$. It follows from (14) and (15), that $\mathcal{E}_{\lambda}$ is invariant for $S_{\lambda}$ and $S_{\lambda}^{*}$, and $T \stackrel{\text { df }}{=} \overline{\left(\left.S_{\lambda}\right|_{\mathcal{E}_{\lambda}}\right)}$ is a creation operator in $\overline{\mathcal{E}}_{\lambda}$. Since $\mathcal{E}_{\lambda}$ is a core for $T^{*}$ (cf. [6, (1.11)]) and $\left.T^{*}\right|_{\mathcal{E}_{\lambda}} \subset S_{\lambda}^{*}$, we get $T^{*} \subset S_{\lambda}^{*}$. Hence $(T+\lambda I)^{*} \subset S_{\lambda}^{*}+\bar{\lambda} I=S^{*}$ and finally $\mathcal{N}\left((T+\lambda I)^{*}\right) \subset \mathcal{N}\left(S^{*}\right)=\mathbb{C} \cdot e_{0}$. However $\operatorname{dim} \mathcal{N}\left((T+\lambda I)^{*}\right)=1$ because $T$ is a creation operator. Thus $e_{0} \in \mathcal{N}\left((T+\lambda I)^{*}\right)$. Replacing $\lambda, S$ and $f_{0}(\lambda)$ by $-\lambda, T$ and $e_{0}$, respectively, we conclude from $T \subset S_{\lambda}$ that $\mathcal{D}_{\boldsymbol{e}}=\operatorname{lin}\left\{S^{n} e_{0} ; n \geq 0\right\}=\operatorname{lin}\left\{(T+\lambda I)^{n} e_{0} ; n \geq 0\right\} \subset \mathcal{D}(T+\lambda I) \subset \mathcal{D}(S)$. Since $\mathcal{D}_{\boldsymbol{e}}$ is a core for $S$, we have $S=\overline{\left.S\right|_{\mathcal{D}_{e}}}=\overline{\left.(T+\lambda I)\right|_{\mathcal{D}_{e}}} \subset T+\lambda I \subset S$. Hence $S_{\lambda}=T$ is a creation operator, which completes the proof.

REMARK 5. The way Theorem 4 is stated may suggest that the operator $S$ becomes a creation one with respect to the same basis as it is assumed to be a weighted shift. We have to point out that this need not be the case. The only thing we get is that these two bases must necessarily be related each to the other as in Proposition 1. However, if $S$ is assumed to be a weighted shift with respect to an orthonormal basis $\left\{e_{n}\right\}_{n=0}^{\infty}$ (the input basis, say) with positive weights $\left\{\sigma_{n}\right\}_{n=0}^{\infty}$ and $\sigma_{0}=1$, then-according to the proof of (ii) $\Rightarrow$ (iii) of Theorem 4 - the basis with respect to which $S$ becomes a creation operator (the output one) coincides with $\left\{e_{n}\right\}_{n=0}^{\infty}$.

COROllary 6. Let $S$ be a closed operator in a Hilbert space $\mathcal{H}$. Then the conditions (i) and (iii) of Theorem 4 are equivalent to

(ii') there exist $\lambda_{1}, \lambda_{2} \in \mathbb{C}, \lambda_{1} \neq \lambda_{2}$ such that the operators $S-\lambda_{1} I$ and $S-\lambda_{2} I$ are weighted shifts.

Comment. Similar results for the annihilation operator $a^{-}$can be easily deduced from Theorem 4 and Corollary 6 . They can be formulated just by replacing "weighted shift" by "backward weighted shift" and "creation" by "annihilation" in Theorem 4 and Corollary 6.

4. The Segal-Bargmann space. An immediate interpretation of Theorem 4 can be realized in the context of the Segal-Bargmann space $\mathcal{A}^{2}\left(\pi^{-1} \exp \left(-|z|^{2}\right) d x d y\right)$ which is composed of all entire functions in $\mathcal{L}^{2}\left(\pi^{-1} \exp \left(-|z|^{2}\right) d x d y\right)(\operatorname{cf}$. [12,2]). Let $S$ be an operator in $\mathcal{A}^{2}\left(\pi^{-1} \exp \left(-|z|^{2}\right) d x d y\right)$ such that $S\left(Z^{n}\right)=\omega_{n} Z^{n+1}$ with $\omega_{n}$ being positive, $n=0,1, \ldots$, and $\omega_{0}=1$. If for some $\lambda \neq 0$ the operator $S-\lambda I$ is a weighted shift, then it can be deduced from Theorem 4 and Remark 5 that $S$ must 
necessarily multiply polynomials by the independent variable $Z$ (i.e., $\omega_{n} \equiv 1$ ), provided the polynomials constitute a core for $S$.

5. The Heisenberg couple. Given an orthonormal basis $\boldsymbol{e}=\left\{e_{n}\right\}_{n=0}^{\infty}$ in $\mathcal{H}$ and a sequence of nonzero complex numbers $\boldsymbol{\sigma}=\left\{\sigma_{n}\right\}_{n=0}^{\infty}$, denote by $\mathfrak{\Im}(\boldsymbol{e}, \boldsymbol{\sigma})$ the set of all pairs $(Q, P)$ of closed symmetric operators in $\mathcal{H}$ such that

$$
\begin{aligned}
\mathcal{D}_{\mathbf{e}} & =\operatorname{lin}\left\{e_{n} ; n \geq 0\right\} \subset \mathcal{D}(Q) \cap \mathcal{D}(P), \\
Q e_{n} & =\bar{\sigma}_{n-1} e_{n-1}+\sigma_{n} e_{n+1} \quad n \geq 0, \\
P e_{n} & =i\left(\bar{\sigma}_{n-1} e_{n-1}-\sigma_{n} e_{n+1}\right) \quad n \geq 0,
\end{aligned}
$$

where, by definition, $\sigma_{-1}=0$ and $e_{-1}=0$. In other words, the restrictions of $Q$ and $P$ to $\mathcal{D}_{\boldsymbol{e}}$ have Jacobi matrix representations

$$
\begin{gathered}
\left.Q\right|_{\mathcal{D}_{e}}=\left(\begin{array}{ccccc}
0 & \bar{\sigma}_{0} & 0 & 0 & \ldots \\
\sigma_{0} & 0 & \bar{\sigma}_{1} & 0 & \ldots \\
0 & \sigma_{1} & 0 & \bar{\sigma}_{2} & \ldots \\
0 & 0 & \sigma_{2} & 0 & \ldots \\
\vdots & \vdots & \vdots & \vdots & \ddots
\end{array}\right), \\
\left.P\right|_{\mathcal{D}_{e}}=\left(\begin{array}{ccccc}
0 & i \bar{\sigma}_{0} & 0 & 0 & \ldots \\
-i \sigma_{0} & 0 & i \bar{\sigma}_{1} & 0 & \ldots \\
0 & -i \sigma_{1} & 0 & i \bar{\sigma}_{2} & \ldots \\
0 & 0 & -i \sigma_{2} & 0 & \ldots \\
\vdots & \vdots & \vdots & \vdots & \ddots
\end{array}\right) .
\end{gathered}
$$

Set $\mathfrak{S}=\bigcup_{\boldsymbol{e}, \boldsymbol{\sigma}} \mathfrak{S}(\boldsymbol{e}, \boldsymbol{\sigma})$, where the union is taken over all possible pairs of $\boldsymbol{e}$ 's and $\boldsymbol{\sigma}$ 's.

Lemma 7. If $S$ and $T$ are symmetric operators in $\mathcal{H}$ such that $\mathcal{D}_{\boldsymbol{e}} \subset \mathcal{D}(S) \cap \mathcal{D}(T)$ and $\left.S\right|_{\mathcal{D}_{e}}=\left.T\right|_{\mathcal{D}_{e}}$, then $\left.S\right|_{\mathcal{D}_{\boldsymbol{e}}} \subset T \subset\left(\left.S\right|_{\mathcal{D}_{e}}\right)^{*}$. If $(Q, P) \in \mathfrak{S}(\boldsymbol{e}, \boldsymbol{\sigma})$, then there exists $(\tilde{\boldsymbol{e}}, \tilde{\boldsymbol{\sigma}})$ such that $(Q, P) \in \mathfrak{S}(\tilde{\boldsymbol{e}}, \tilde{\boldsymbol{\sigma}})$ and $\tilde{\sigma}_{n}>0$, for every $n \geq 0$.

Proof. Taking adjoints over $\left.T\right|_{\mathcal{D}_{e}} \subset T$ yields $\left.S\right|_{\mathcal{D}_{e}}=\left.T\right|_{\mathcal{D}_{e}} \subset T \subset T^{*} \subset\left(\left.T\right|_{\mathcal{D}_{e}}\right)^{*}=$ $\left(\left.S\right|_{\mathcal{D}_{e}}\right)^{*}$. Set $\tilde{e}_{0}=e_{0}, \tilde{e}_{n+1}=\sigma_{0} \sigma_{1} \cdots \sigma_{n}\left|\sigma_{0} \sigma_{1} \cdots \sigma_{n}\right|^{-1} e_{n+1}$ and $\tilde{\sigma}_{n}=\left|\sigma_{n}\right|$ for $n \geq 0$. Then the pair $(\tilde{\boldsymbol{e}}, \tilde{\boldsymbol{\sigma}})$ meets our requirements.

We say that $(Q, P)$ is a Heisenberg couple (with respect to an orthonormal basis $\boldsymbol{e}$ for $\mathcal{H})$ if $(Q, P) \in \mathfrak{S}(\boldsymbol{e}, \boldsymbol{\sigma})$ with $\sigma_{n}=\sqrt{\frac{n+1}{2}},(n \geq 0)$. Heisenberg couples are characterized (up to a multiplicative constant) by the following invariance property.

THEOREM 8. If $(Q, P) \in \mathfrak{S}$, then the following conditions are equivalent:

(i) for all $\xi, \eta \in \mathbb{R},(Q+\xi I, P+\eta I) \in \mathfrak{\Im}$,

(ii) there exist $\xi, \eta \in \mathbb{R}$ such that $\xi^{2}+\eta^{2}>0$ and $(Q+\xi I, P+\eta I) \in \mathfrak{J}$,

(iii) there exists $\alpha \in \mathbb{R} \backslash\{0\}$ such that $(\alpha Q, \alpha P)$ is a Heisenberg couple.

Moreover, if $(Q, P)$ is a Heisenberg couple, then $(Q+\xi I, P+\eta I)$ is a Heisenberg couple for all $\xi, \eta \in \mathbb{R}$. 
Proof. (ii) $\Rightarrow$ (iii) By Lemma 7 there exists $(\boldsymbol{e}, \boldsymbol{\sigma})$ such that $(Q, P) \in \mathfrak{S}(\boldsymbol{e}, \boldsymbol{\sigma})$ and $\sigma_{n}>0$ for $n \geq 0$. Denote by $S$ the closed weighted shift with respect to the basis $\boldsymbol{e}$ with weights $\boldsymbol{\sigma}$. It is clear that $\left.2 S\right|_{\mathcal{D}_{e}}=\left.Q\right|_{\mathcal{D}_{e}}+\left.i P\right|_{\mathcal{D}_{e}}$. This, [6, (1.13)] and Lemma 7 imply $2 S=\overline{\left.Q\right|_{\mathcal{D}_{e}}+\left.i P\right|_{\mathcal{D}_{e}}} \subset \overline{Q+i P} \subset \overline{\left(\left.Q\right|_{\mathcal{D}_{e}}\right)^{*}+i\left(\left.P\right|_{\mathcal{D}_{e}}\right)^{*}}=2 S$. Hence

$$
S=\frac{1}{2}(\overline{Q+i P})
$$

Let $\left(\boldsymbol{e}^{\prime}, \boldsymbol{\sigma}^{\prime}\right)$ be such that $(Q+\xi I, P+\eta I) \in \mathfrak{J}\left(\boldsymbol{e}^{\prime}, \boldsymbol{\sigma}^{\prime}\right)$. Denote by $S^{\prime}$ the weighted shift with respect to $\boldsymbol{e}^{\prime}$ with weights $\boldsymbol{\sigma}^{\prime}$. Set $\lambda=\frac{1}{2}(\xi+i \eta)$. Analysis similar to that in the proof of (16) shows that $S^{\prime}=\frac{1}{2}(\overline{(Q+\xi I)+i(P+\eta I)})$. This and (16) give us $S^{\prime}=\frac{1}{2}(\overline{Q+i P})+\lambda I=S+\lambda I$, which means that $S+\lambda I$ is a weighted shift. It follows from the proof of (ii) $\Rightarrow$ (iii) of Theorem 4 that $\sigma_{n}=\sigma_{0} \sqrt{n+1}$ for $n \geq 0$ (because $\lambda \neq 0)$. Thus $(\alpha Q, \alpha P)$ is a Heisenberg couple with respect to $\boldsymbol{e}$ with $\alpha=\left(\sqrt{2} \sigma_{0}\right)^{-1}$.

(iii) $\Rightarrow$ (i) Without any loss of generality we can assume that $\alpha=1$. Suppose that $(Q, P)$ is a Heisenberg couple with respect to an orthonormal basis $\boldsymbol{e}$. Let $S_{0}$ be a creation operator with respect to $\boldsymbol{e}$. Set $S=\frac{1}{\sqrt{2}} S_{0}$. Since $\overline{\left.Q\right|_{\mathcal{D}_{\boldsymbol{e}}}}$ is selfadjoint, $Q$ is symmetric and $\overline{S+S^{*}}=\overline{\left.Q\right|_{\mathcal{D}_{e}}} \subset Q$ (cf. [6, (2.2)]), we get $Q=\overline{\left.Q\right|_{\mathcal{D}_{e}}}=\overline{S+S^{*}}$. Likewise, we show that $P=\overline{\left.P\right|_{\mathcal{D}_{e}}}=-i\left(\overline{S-S^{*}}\right)$. Take two real numbers $\xi, \eta$ and define $\lambda=\frac{1}{2}(\xi+i \eta)$. It follows from Theorem 4 that $\sqrt{2}(S+\lambda I)=S_{0}+\sqrt{2} \lambda I$ is a creation operator with respect to an orthonormal basis $\boldsymbol{e}^{\prime}$. Therefore $\left(Q_{\lambda}, P_{\lambda}\right) \stackrel{\mathrm{df}}{=}$ $\left(\overline{(S+\lambda I)+(S+\lambda I)^{*}},-i\left(\overline{(S+\lambda I)-(S+\lambda I)^{*}}\right)\right)$ is a Heisenberg couple with respect to $\boldsymbol{e}^{\prime}$ (cf. [6, Section 2]). This and the equality $(Q+\xi I, P+\eta I)=\left(Q_{\lambda}, P_{\lambda}\right)$ complete the proof.

REMARK 9. It follows from the proof of (ii) $\Rightarrow$ (iii) of Theorem 8 that the observation made in Remark 5 applies here as well. More precisely, $\sigma_{0}=\frac{1}{\sqrt{2}}$ and $\sigma_{n}>0$ for $n=1,2, \ldots$ in the input basis make it coincide with the output one.

REMARK 10. It is worthwhile to notice that the definition of $\mathfrak{S}(\boldsymbol{e}, \boldsymbol{\sigma})$ does not require $\mathcal{D}_{\boldsymbol{e}}$ to be a core of either $Q$ or $P,(Q, P) \in \mathfrak{S}(\boldsymbol{e}, \boldsymbol{\sigma})$, and this is maintained by Theorem 8 as well. On the other hand, it follows from the proof of Theorem 8 that if $(Q, P)$ is a Heisenberg couple with respect to an orthonormal basis $\boldsymbol{e}$, then $Q=\overline{\left.Q\right|_{\mathcal{D}_{e}}}$, $P=\overline{\left.P\right|_{\mathcal{D}_{e}}}$ and both the operators $Q$ and $P$ are selfadjoint. Thus, starting in Theorem 8 with a pair of symmetric operators $Q$ and $P$ we conclude their selfadjointness by the way.

It might be interesting to remind the reader here that (see [6, Section 2]) the operators $Q$ and $P$ are unitarily equivalent.

6. The Schrödinger couple. Though the Schrödinger couple, as a unitary image of the Heisenberg one in $\mathcal{L}^{2}(\mathbb{R})$, behaves in the same way we would like to display this case in more detail. Recall that the Hermite functions $h_{n}, n=0,1, \ldots$, are defined as

$$
h_{n}(x) \stackrel{\mathrm{df}}{=} 2^{-n / 2}(n !)^{-1 / 2} \pi^{-1 / 4} e^{-x^{2} / 2} H_{n}(x) \quad(x \in \mathbb{R}),
$$

with $H_{n}$, the $n$-th Hermite polynomial,

$$
H_{n}(x) \stackrel{\mathrm{df}}{=}(-1)^{n} e^{x^{2}} \frac{d^{n}}{d x^{n}} e^{-x^{2}} \quad(x \in \mathbb{R}),
$$


and they form an orthonormal basis in $\mathcal{L}^{2}(R)$. The pair of selfadjoint operators

$$
\left(x, i \frac{d}{d x}\right) \text { belongs to } \mathfrak{\Im}\left(\left\{h_{n}\right\}_{n=0}^{\infty},\{\sqrt{(n+1) / 2}\}_{n=0}^{\infty}\right)
$$

and is known as the Schrödinger couple.

Let us start with a couple $(Q, P)$ of symmetric operators in $\Im\left(\left\{h_{n}\right\}_{n=0}^{\infty}, \boldsymbol{\sigma}\right)$, where $\boldsymbol{\sigma}=\left\{\sigma_{n}\right\}_{n=0}^{\infty}$ is a sequence of positive real numbers with $\sigma_{0}=\frac{1}{\sqrt{2}}$. If we suppose that for some $\xi, \eta \in \mathbb{R}$ with $\xi^{2}+\eta^{2}>0$ the couple $(Q+\xi I, P+\eta I)$ is in $\mathfrak{I}$, then from Theorem 8, in view of Remark 9, we get

$$
(Q, P) \text { belongs to } \mathfrak{\Im}\left(\left\{h_{n}\right\}_{n=0}^{\infty},\{\sqrt{(n+1) / 2}\}_{n=0}^{\infty}\right) .
$$

Comparing this with (17) we can identify $Q$ as $x$ and $P$ as $i \frac{d}{d x}$ (use Remark 10).

\section{REFERENCES}

1. W. Arveson, D. Hadwin, T. B. Hoover and E. Kymala, Circular operators, Indiana Univ. Math. J. 33 (1984), 583-595.

2. V. Bargmann, On a Hilbert space of analytic functions and an associated integral transform, Comm. Pure Appl. Math. 14 (1961), 187-214.

3. J. Beckers, N. Debergh and F. H. Szafraniec, A proposal of new sets of squeezed states, Phys. Lett. A, 243 (1998), 256-260.

4. J. Beckers, N. Debergh and F. H. Szafraniec, On oscillatorlike Hamiltonians and squeezing, Int. J. Theoretical Physics 36 (2000), 1515-1527.

5. W. Mlak, Notes on quantum circular operators, Institute of Mathematics, Polish Academy of Sciences, preprint \# 303, 1984.

6. W. Mlak, The Schrödinger type couples related to weighted shifts, Univ. Iagel. Acta Math. 27 (1988), 297-301. 307.

7. W. Mlak, Note on unitary circular operators, Univ. Iagel. Acta Math. 27 (1988), 303-

8. W. Mlak, Notes on circular operators I, II, III, IV, Univ. Iagel. Acta Math. 29 (1992), 145-152, 153-162, 163-170, 171-175.

9. W. Mlak and M. Słociński, Quantum phase and circular operators, Univ. Iagel. Acta Math. 24 (1992), 133-144.

10. W. Mlak and J. Stochel, A note on coherent states related to weighted shifts, Publ. RIMS, Kyoto Univ. 28 (1992), 587-593.

11. C. R. Putnam, Commutation properties of Hilbert space operators and related topics (Springer Verlag, 1967).

12. I. E. Segal, Mathematical problems of relativistic physics, Chap. IV in Proceedings of the Summer Seminar, Boulder, Colorado 1960, vol II, ed. M. Kac; Lectures in Applied Mathematics (AMS, Providence, RI, 1963).

13. A. L. Shields, Weighted shift operators and analytic function theory, Math. Surveys 13 (1974), 49-128.

14. Jan Stochel and F. H. Szafraniec, On normal extensions of unbounded operators. III. Spectral properties, Publ. RIMS, Kyoto Univ. 25 (1989), 105-139.

15. Jan Stochel and F. H. Szafraniec, A few assorted questions about unbounded subnormal operators, Univ. Iagel. Acta Math. 28 (1991), 163-170.

16. Jerzy B. Stochel, Subnormality and generalized commutation relations, Glasgow Math. J. 30 (1988), 259-262.

17. F. H. Szafraniec, Yet another face of the creation operator, Operator Theory $A d v$. Appl. 80 (1995), 266-275.

18. F. H. Szafraniec, Analytic models of the quantum harmonic oscillator, Contemp. Math. 212 (1997), 269-276. 
19. F. H. Szafraniec, The quantum harmonic oscillator in $\mathcal{L}^{2}(R)$, in Special Functions and Differential Equations, Proceedings, Madras (India), January 13-24, 1997, eds. K. Srinivasa Rao, R. Jagannathan, G. Vanden Berghe, J. Van der Jeugt, pp. 206-211, Allied Publishers, New Dehli, 1997.

20. F. H. Szafraniec, An inductive limit procedure within the quantum harmonic oscillator, Operator Theory Adv. Appl., 106 (1998), 389-395.

21. F. H. Szafraniec, Subnormality in the quantum harmonic oscillator, Comm. Math. Phys. 210 (2000), 323-334.

22. W. Szymański, Circular operators related to some quantum observables, Ann. Polon. Math. 66 (1997), 253-261.

23. H. G. Tillmann, Zur Eindeutigkeit der Lösungen der quantummechanischen Vertauschungsrelationen, Acta Sci. Math. (Szeged) 24 (1963), 258-270. 